\title{
Hunger level during food consumption: Effects on subsequent preference
}

\author{
SAMUEL H. REVUSKY \\ BIOPHYSICS DIVISION, US ARMY MEDICAL RESEARCH LABORATORY, FORT KNOX, KENTUCKY
}

\begin{abstract}
Rats in Group $G$ were fed grape juice while hungry and milk while satiated; Group $M$ was fed milk while hungry and grape juice while satiated. In a subsequent choice between grape juice and milk, Group $G$ preferred grape juice more than Group $M$. It is theorized that the mechanism responsible for this change in preference is also largely responsible for the major effects of hunger on performance.
\end{abstract}

Psychologists have tended to use one methodology to observe the behavioral effects of the deprivation of all food and another methodology for dietary deficiencies. Increasing deprivation is usually observed to increase the frequency of food rewarded responses while an increased dietary deficiency is usually observed to increase the preference for the needed nutrient. It is possible to measure the effects of dietary deficiencies by the procedures usually used for generalized food deprivation; for instance, parathyroidectomized rats will work harder for food containing calcium as a function of hours of calcium deprivation (Lewis, 1964). The present report describes an attempt to measure the effects of deprivation by a preference technique usually used for dietary deficiency. This is the experimental strategy. One group of rats (called Group G) is fed grape juice under 20.5 hr. food deprivation and milk while nearly satiated; Group $M$ is fed milk under deprivation and grape juice while satiated. Both groups are later allowed to choose between grape juice and milk. If Group G prefers grape juice more than Group $M$, it will be inferred that the preference for a food increases with the deprivation time at which it has previously been ingested, just as the preference for a needed nutrient increases with increased deficiency. If so, generalized hunger and specific hungers are best explicable by similar underlying processes.

Method

Both Group G and Group $M$ contained 12 male and 12 female Long Evans rats, between 60 and 90 days old. During the entire duration of the experiment and for at least seven days prior to it, they were given free access to Teklad rat meal for $75 \mathrm{~min}$. a day. Preference training was administered in the home cage for five days. For Group $\mathrm{G}$, it consisted of leaving $5 \mathrm{ml}$ of Welch grape juice in a food cup $135 \mathrm{~min}$. prior to the Teklad feeding; shortly after the Teklad feeding, $5 \mathrm{ml}$ of Pet evaporated milk was similarly administered. Group $M$ received the counterbalanced procedure. Preference testing began two days after preference training. It consisted of $30 \mathrm{~min}$. in a cage similar to the home cage which contained two drinking bottles; the left bottle contained grape juice and the right bottle contained milk. To insure generality, a subgroup of each group, three males and three females, was tested at each of four deprivation times: $0,3,9$, and $23 \mathrm{hr}$. Preference for grape juice was defined as the number of licks to the spout containing grape juice divided by the total number of licks to both spouts (measured by drinkometers).

\section{Results}

Although neither group preferred grape juice to milk, the mean preference for grape juice relative to milk in Group $G$ was .29, which was higher than the .14 preference in Group M. An F test (groups by test deprivation) yielded $p<.002$ for groups, $p>.25$ for test deprivation, and $p<.05$ for the interaction. The just barely significant interaction does not mean that the difference between Group $G$ and Group $M$ disappeared at some test deprivation levels because one-tailed $t$ tests comparing the two groups at a single deprivation level yielded significant results $(\mathrm{p}<.01)$ at 0 and $9 \mathrm{hr}$. and a nearly significant result at $23 \mathrm{hr} .(p<.10)$. Thus preference for a food was greater if the food had previously been consumed at a high deprivation level than at a low deprivation level regardless of the test deprivation. (If the significant interaction is not a sampling error, it means that the difference between the two groups was greatest at a $0 \mathrm{hr}$. test deprivation level, but the present experiment did not yield adequate data to show minor changes in preference as a function of test deprivation since that was not its purpose.)

\section{Discussion}

Smith \& Capretta (1956) have shown that rats, rewarded with saccharin solution for making a correct $\mathrm{T}$-maze response, make more errors if they have previously consumed saccharin solution under high deprivation than if they have consumed it under low deprivation. Thus, it may be inferred that the preference for nonnutritive foods decreases as a function of the deprivation level at which they have been ingested in the past; this is the opposite of the result reported here for nutritive foods. Consequently, I believe that the present result was due to delayed nutritional aftereffects which become more rewarding as deprivation increases. Aftereffects which occur long after ingestion can affect food preference as follows. If ingestion of a food is followed, as much as a number of hours later, by radiation sickness (Kimeldorf \& Hunt, 1965) or by injection of poison (Garcia, Ervin, \& Koelling, 
1966), preference for that food will be reduced. Thiamine injection some minutes after saccharin consumption increases the preference of thiamine deficient rats for saccharin (Garcia, Yorke, Ervin, \& Koelling, unpublished data).

Changes in taste preferences produced by the delayed aftereffects of ingestion are probably responsible for bait-shyness (Garcia, Ervin, \& Koelling, 1966) and the specific hunger for foods containing thiamine (Harris, Clay, Hargreaves, \& Ward, 1933; Scott \& Verney, 1947). It is conjectured here that delayed aftereffects of ingestion also become associated with drive stimuli and that this association, rather than any association with tastes, is responsible for the usual effects of food deprivation on food-motivated performance. The details are as follows. Drive stimuli (internal stimuli corresponding to the animal's deprivation state) exist because a rat can select one of two responses when the only cue supplied is deprivation time (Kimble, 1961). It is assumed that they can be associated with the delayed aftereffects of ingestion just as tastes can. It is known from operant conditioning experiments that if a response produces a larger amount of reward (Keesey \& Kling, 1961) or a sweeter reward (Goodrich, 1965) in the presence of $S_{1}$ than in the presence of $S_{2}$, rate of response will be higher in the presence of $S_{1}$. Analogously, it is conjectured that the amount of food an animal will eat and the amount of food-motivated work an animal will do will be greater in the presence of high drive stimuli than low drive stimuli because the delayed aftereffects of food ingestion will be more rewarding. The present experiment gave tastes the relationships to deprivation level which drive stimuli usually have; thus the preference for a food increased when its taste had previously been associated with high deprivation.

If conditioning of drive stimuli to the delayed aftereffects of ingestion is responsible for all the motivational effects of deprivation on performance, it must account for the fact that the effect of a change in deprivation level on performance is likely to be immediate. For instance, if rats trained under a single deprivation level, are divided into groups and extinguished at a variety of deprivation levels, rate of response is an increasing function of deprivation level (Kimble, 1961) even though the rats have had no opportunity to learn a drive stimulus discrimination in the test situation. Thus, if the present conjectures are correct, a discrimination which rats have learned in the past must be able to affect performance in extinction even if there is no discriminative training in the test situation. Such transfers of simple discriminations have been demonstrated (Estes, 1948; Morse \& Skinner, 1958). It is conjectured that the more complex discrimination hypothesized here can similarly be transferred, both because there a re innate factors increasing the predisposition to learn such a discrimination (Garcia \& Koelling, 1966) and because the animals have very extensive experience with it in the course of their daily feeding cycles. If so, drive stimuli are able to function as trans-situational motives.

As far as I can see, the proposed mechanism is compatible with all the known facts about generalized hunger, although this does not guarantee that no other factors are involved. In contrast to theories which claim unsubstantiated properties for the drive stimulus dimension or postulate mysterious activations of the animal produced by deprivation, the proposed view depends entirely on extrapolations from known facts; admittedly, analogies are used to more than a desirable extent. Finally, the principles used here to explain generalized hunger dovetail with principles usually used to explain specific hungers and amount of reinforcement effects; this seems reasonable because all these effects are quite similar.

\section{References}

Estes, W. K. Discriminative conditioning II. Effects of a Pavlovian conditioned stimulus upon a subsequently established operant response. J. exp. Psychol., 1948, 38, 173-177.

Garcia, J., Ervin, F. R., \& Koelling, R. A. Learning with prolonged delay of reinforcement. Psychon. Sci., 1966, 5, 121-122.

Garcia, J., \& Koelling, R. A. Relation of cue to consequence in avoidance learning. Psychon. Sci., 1966, 4, 123-124.

Goodrich, K. P. Differential conditioning based on concentration of sucrose reinforcement in rats on a VI schedule. Psychon. Sci., $1965,3,213-214$.

Harris, L. J., Clay, J., Hargreaves, F. J., \& Ward, A. Appetite and choice of diet: The ability of the vitamin B deficient rat to discriminate between diets containing and lacking the vitamin. Proc. Roy. Soc., London B, 1933, 113, 161-190.

Keesey, R. E., \& Kling, J. W. Amount of reinforcement and free operant responding. J. exp. Anal. Behav., 1961, 3, 125-132.

Kimble, G. A. Hilgard and Marquis' conditioning and learning. New York: Appleton-Century-Crofts, 1961.

Kimeldorf, D. J., \& Hunt, E. L. Ionizing radiation: neural function and behavior. New York: Academic Press, 1965.

Lewis, Melissa. Behavior resulting from calcium deprivation in parathyroidectomized rats. J. comp. physiol. Psychol., 1964, $57,348-352$.

Morse, W. H., \& Skinner, B. F. Some factors involved in the stimulus control of operant behavior. J. exp. Anal. Behav., 1958, 1, 103- 107 .

Scott, E. M., \& Vemey, Ethel L. Self selection of diet VI: the nature of appetites for B vitamins. J. Nutrition, 1947, 34, 471480 .

Smith, M. P., \& Capretta, P. J. Effects of drive level and experience on the reward value of saccharine solutions. $J$. comp. physiol. Psychol., 1954, 49, 553-557. 